\title{
Physico-chemical characterization of hybridized graphene and boron-nitride layers
}

\author{
Paulina Raquel Martínez-Alanis ${ }^{1}$, Antonio Alvarez de la Paz ${ }^{2}$, Ruben Santamaria ${ }^{2, *}$ \\ ${ }^{1}$ Depart. Química Inorgànica i Orgànica, Secc. Química Inorgànica, Fac. Química, Universitat de Barcelona, \\ Martí i Franqués, 1-11, 08028, Barcelona, Spain \\ ${ }^{2}$ Depto. de Física Teórica, Instituto de Física, UNAM, Cd. Mex., México
}

\begin{abstract}
New materials can be created by modifying matter. In this work, we characterize graphene and boronnitride (BN) layers after doping them with $\mathrm{BN}$ and carbon dimers, respectively, in different amounts and with different spatial distributions. We provide the energetic description, electron density features, molecular electrostatic potential maps, net charge populations, and the speeds of propagating waves on the hybridized layers. We show the possibility of designing molecular electrostatic potentials from a spatially controlled doping. A strategy is illustrated on a BN hybrid layer with the adsorption of DNA nucleic acid bases.
\end{abstract}

Keywords: graphene layer, boron nitride sheet, doping effects, molecular characterization, density functional theory.

\section{Introduction}

The graphene and the boron nitride are 2D materials described as hexagonal lattices with only $1.8 \%$ of lattice spacing difference ${ }^{1-5}$. Still, they own different chemical and physical properties; the graphene layer is a conductor, while the boron nitride layer is an insulator ${ }^{6,7}$. The graphene is known to be a remarkable material with unique chemical, thermal ${ }^{8,9}$, electrical ${ }^{5}$, and mechanical properties ${ }^{10}$. For example, it has a thermal conductivity of 2000-4000 $\mathrm{W} / \mathrm{mK}$ for freely suspended samples ${ }^{9}$, an elastic modulus of $1 \pm 0.1 \mathrm{Tpa}^{11,12}$ showing a strength that makes graphene one of the strongest material ever measured, ${ }^{11}$ and with ultrastrong adhesion ${ }^{12}$, among other features. Such properties make graphene a promising material for applications in electronic devices ${ }^{13}$, electrochemical energy storage ${ }^{14}$, and biomaterials ${ }^{15}$ at the nanoscale regime. The graphene is presented as a mono-layer or multi-layer material. The common methods employed to obtain graphene are the chemical vapor deposition ${ }^{16,17}$, chemical synthesis sonication 18 , and micromechanical exfoliation ${ }^{19}$. The fabrication method determines the number of layers that can be created in the material, and its mechanical properties ${ }^{10}$. On the other side, the boron nitride layers are chemically stable, exhibit high thermal conductivity concerning that of graphene, and offer resistance to oxidation ${ }^{20}$. A family of boron monolayers has been found to own novel semiconducting properties, in principle attributed to the motif of hexagonal vacancies ${ }^{21}$. The mechanical strength of the boron nitride nanosheets has been found to be indifferent to the increasing thickness, on the number of layers, due to the interlayer interactions which are different to those occurring in graphene ${ }^{22}$. It has also been theoretically and experimentally shown that boron nitride nanosheets suffer conformational changes with the adsorption of molecules, with a resultant increase of adsorption energy and efficiency ${ }^{23}$.

In spite of their different chemical and physical properties, the boron nitride and graphene layers have the same hexagonal structure: the bond length of boron nitride is on average $1.44 \AA$, and that of CC in graphene is on average $1.42 \AA{ }^{24}$ Due to the lattice similarities between the graphene and boron nitride layers, it has been possible to produce composite materials. For example, bilayer graphene samples sandwiched between layers of hexagonal boron nitride were created with the goal of tuning excitons (this is, bound states of an electron and a hole), aiming at optoelectronic applications ${ }^{25}$. On the other side, $\mathrm{Ci}$ et al. mixed domains of boron nitride with graphene, controlling the amount of carbon from $10 \%$ to $100 \%{ }^{26}$. The hybridization of the three elements produced mixed atomic layers, which were confirmed using the EELS technique. The studies with XPS in the same work also exhibited shoulders at a lower binding energy for B (due to the electronegativity of $\mathrm{C}$ ), and at higher energy for $\mathrm{N}$ (due to the bonding with $\mathrm{C}$ ). The authors measured lower optical band gaps by increasing the concentration of $\mathrm{C}$. 
The optical band gap measured by ultravioletvisible absorption spectra of the first absorption edge on h-BNC at $65 \% \mathrm{C}$ was $4.48 \mathrm{eV}$, and even lower when increasing the percentage of $\mathrm{C}$ to $84 \%$ with a value of $3.85 \mathrm{eV}$, in comparison to the $5.69 \mathrm{eV}$ band gap of pure h-BN. Similar behavior was obtained for the second absorption edge by increasing the quantity of $\mathrm{C}$ in the h-BNC layer. These experiments show the possibility to tune the electronic properties of mixed monoatomic surfaces by the content of BN-C elements. The experiments, in combination with theoretical models, explain the electronic properties of the band gap behavior. Nascimento et al. calculated the DFT energy band gaps of $\mathrm{BxCyNx}$ structures using Monte-Carlo absorption temperature hopping, in different concentrations of the randomly positioned elements ${ }^{27}$. They equally observed lower band gaps values when increasing the $\mathrm{C}$ content in different $\mathrm{BNC}$ structures. In the same study, the authors determined the mechanical behavior of uni-axial strain effects in the monoatomic mixed layers in both the zigzag and armchair directions varying the band gap energy of a B5C86N5 structure. Recent work has been focused on the graphene lattice doped with boron nitride $(\mathrm{BN})$ for applications on water purification ${ }^{28}$. In short, the electrical properties of a graphene layer can be modified by replacing pairs of carbon atoms by boron nitride compounds 26,29 and, in principle, the modification may be performed by gradually doping the graphene layer with $\mathrm{BN}$, until reaching the total transformation of the graphene sheet onto a pure boron nitride sheet, and vice versa ${ }^{30}$. In general, the studies have been focused on investigating the semiconducting properties for band gap-engineered applications in electronics and optics ${ }^{26}$. Several questions referent to the physical and chemical properties in the interconversion of both materials persist. In this respect, it is of interest, for example, to evaluate the mechanical and chemical changes caused by the BN doping of a graphene lattice.

The purpose of this work is to explore the distinctive features between a graphene layer and its doped boron nitride derivatives, and vice versa, from a physico-chemical perspective. We start with a pristine graphene lattice of the rectangular shape formed by 142 atoms, where 28 atoms are hydrogens at the borders that saturate the carbon dangling bonds. By systematically replacing pairs of carbon atoms, $\mathrm{CC}$, with BN compounds in the graphene (gp) lattice, the gp layer is gradually doped with small amounts of BN compounds. Note that, in principle, the doping of graphene can be performed by replacing single carbon atoms with individual boron or individual nitrogen atoms. Nevertheless, our interest resides on doping graphene by replacing $\mathrm{CC}$ pairs by $\mathrm{BN}$ compounds because, in this case, the mass difference between the $\mathrm{CC}$ and $\mathrm{BN}$ dimers reaches a minimum value (more details are given below). On the other hand, by replacing $\mathrm{BN}$ compounds with carbon dimers in a pristine $\mathrm{BN}$ lattice (with equal dimensions to the graphene lattice), the BN layer is gradually hybridized with small amounts of carbons. In principle, such processes allow the interconversion of the grid lattices onto each other. The details of the molecular model are given in the following sections. We pay attention to the energy changes, and examine the electron densities, molecular electrostatic potential maps and charge populations of the gp and $\mathrm{BN}$ layers with different amounts of doping compounds. Also, we briefly focus attention on the molecular vibrations of the gp layers with different amounts of the binary BN compounds. The vibrations of the graphene layer and its $\mathrm{BN}$ derivatives are induced mechanically, by displacing some central atoms of the horizontal planar layer in the vertical direction. The release of such atoms produces a perturbation on the adjacent atoms that propagates in every direction as a wave on the lattice. We use molecular dynamics in combination with density functional theory in this investigation. Previous studies of graphene based materials have used classical molecular dynamics in conjunction with empirical, ${ }^{31,32}$ and ab-initio methods ${ }^{33}$. The method used in this work is sufficiently accurate for the mechanical and energetic description of graphene, but it has the disadvantage of being computationally costly. For such a reason, we employ GPUs in the calculations to reduce the simulation times ${ }^{34}$, more details are given in section 0.2. Summing up, this work contributes to the static and dynamic characterization of gp and BN layers, and their hybridized lattices, by establishing their structural and energetic features at the atomic level, and from a physico-chemical point of view.

\section{Molecular model}

We build in a first stage a graphene layer containing 142 atoms, 114 of them are carbons to form the body's lattice and 28 atoms are hydrogens to saturate the dangling bonds at the borders of the layer (Figure 1).

On the other hand, the $\mathrm{BN}$ dimer is capable to closely adopt the structure of two contiguous carbons in the graphene network. Such a property favors the $\mathrm{BN}$-doping of graphene layers with minimal structural distortions, but simultaneously introducing important chemical changes. Thus, by replacing carbon pairs by $\mathrm{BN}$ compounds, the graphene layer can be gradually doped with, say, 2, 3, 6, and 12 BN compounds (Figures 1 and 2). A pure BN layer (where $57 \mathrm{BN}$ compounds replace the 114 carbons of the graphene mesh) is also included in the investigation. The spatial distribution of the doping compounds goes from small isolated islands to large extended domains in the layers. Once the doping elements are positioned, the layer is energetically optimized and the electron density, molecular electrostatic potential map and atomic charge populations are computed and discussed. The characterization is of static nature as all the atoms remain fixed after acquiring their energetically-optimized positions. With the purpose of additionally introducing a dynamic study, the 
layers are made to vibrate. The vibration is mechanically induced by displacing a few atoms located at the central part of the horizontal-lying layer in the vertical direction. The wave propagation depends of the mesh atomic composition, which in turn determines the mechanical properties of the material, such as the elasticity. For instance, the BN compound has a total atomic mass that differs from that of two carbon atoms (according to the UPAC the atomic mass of $\mathrm{C}$ is 12.01 , that of $\mathrm{B}$ is 10.81 , and the atomic mass of $\mathrm{N}$ is 14.01).

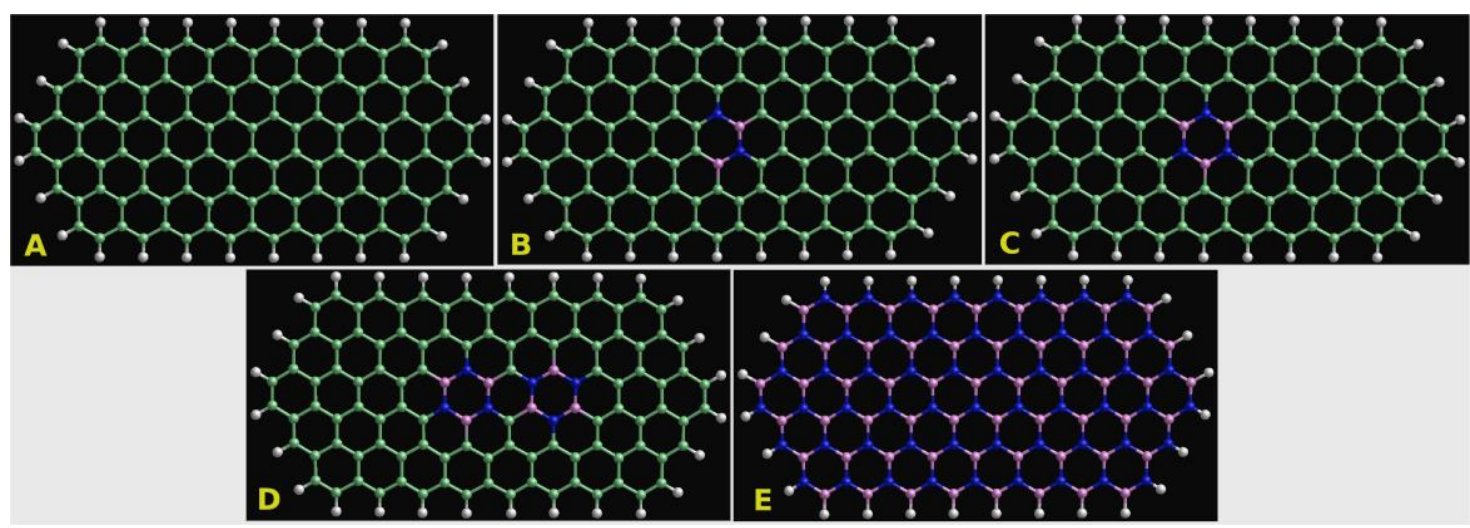

Figure 1. A model layer of graphene is presented in inset A. The carbon bonds are saturated with hydrogens at the borders. The insets B, C and D show the graphene layer doped with 2, 3, and 6BN compounds, respectively.

The inset $\mathrm{E}$ shows the layer where the 114 carbon atoms of inset A are replaced by 57BN compounds (color code: green $=$ carbon, white $=$ hydrogen, blue $=$ nitrogen, pink $=$ boron )

Therefore, the substitution of a couple of carbons by $\mathrm{BN}$ produces a mass difference in the layers. This difference is small in the 142-atom grid, still, the mass difference is expected to play a role in distinguishing the vibrations of a highly BN-doped graphene layer from the vibrations of a pure graphene layer. Boundary conditions are imposed by keeping the hydrogen atoms static in the simulations.

\section{Computational details}

The molecular dynamics method is a powerful simulation tool frequently used in processes where the dynamical character of the particle system is important. In this work we deal with vibrating atoms and, thereby, it is convenient to solve the Newtonian equation of motion following the molecular dynamics method.

$\frac{d p_{i}}{d t}=-\nabla_{i} V\left(\left\{R_{i}\right\} ; t\right)$

The momentum of the ith particle is $p_{i}$ and the interaction potential among the atoms is $V$, which depends on all the atomic coordinates $R_{i}$. In the BornOppenheimer approximation, the electrons move fast, and the nuclei are essentially static. Then, the electrons achieve a stationary state at every configuration of the nuclei. In this context, the interaction potential $V$ is considered the electronic energy of the system (it also includes the Coulomb energy of the nuclei), and we write $V=E$, where $E$ is the electronic energy with parametric dependence on the atomic coordinates. The electronic energy $E$ is obtained after solving the time-independent Schrödinger equation.

$A \psi=E \psi$
His the Hamiltonian operator of the molecular system (where the instantaneous atomic coordinates of Eq. 1 are present), and $\psi$ is the corresponding wave function. We employ the nonlocal version of density functional theory (DFT) to solve the Schroedinger equation. The representations proposed by Becke ${ }^{35}$ and Lee-Yang-Parr ${ }^{36}$ of the exchange and correlation energy functionals are chosen, respectively. The 6$31 \mathrm{G}$ Gaussian basis sets are sufficiently accurate to provide electronic energies and geometries, and they are used in the computations. The Kohn-Sham one electron equations in the DFT method are solved selfconsistently using dynamic grids. The final grid consists of 3,000 points per atom, demanding threshold convergences of $10^{-6}$ au in the density and energy. The DL-FIND optimizer is used to obtain the energy-stable structures ${ }^{37}$. The thresholds imposed on the gradient convergence are max step $=1.8 \times 10^{-3}, \mathrm{rms}$ step $=1.2 \times 10^{-3}$, max grad $=4.5 \times 10^{-4}$ and $\mathrm{rms}$ grad $=$ $3.0 \times 10^{-4}$. On the other side, the Newtonian equations are solved using the velocity-Verlet algorithm with time steps of $1 \mathrm{fs}{ }^{38}$. The calculations of the electron density and molecular electrostatic potential maps are performed using the DFT method.

Our software package solves the equations of motion and is written in the FORTRAN language. It makes system calls to run an external ab-initio program. We take TeraChem ${ }^{39}$ as the external abinitio program because it is written in CUDA language for Nvidia GPU platforms and runs efficiently. Yet, the isosurfaces of the electron density and molecular electrostatic potential are computed at similar level of theory with Gaussian-09 ${ }^{40}$. 


\section{Energy analysis}

The doping of the graphene layer is performed using 2, 3, 6, and 12BN compounds. The small doping cases (with 2, 3 and 6BN dimers) allow establishing the energy changes concerning the BN-doping number (Figure 1). On the other hand, the large doping cases (this is, the different situation with 12BN) allow establishing the energy changes regarding the positions of the $\mathrm{BN}$ doping (Figure 2).
The energy of the bare BN layer is additionally computed. Such a layer is doped with 2, 4 and $8 \mathrm{C}$ atoms. The hybridized lattices represent an isoelectronic series, with the only difference that the nuclei equally distributed in the ${ }^{6} \mathrm{C}-{ }^{6} \mathrm{C}$ dimer have been redistributed in the form ${ }^{5} \mathrm{~B}-{ }^{7} \mathrm{~N}$. Hence, it is of interest to compare their electronic energies. Table 1 provides such energies, as well as the energy differences by taking the energy of the pure graphene layer as the reference energy

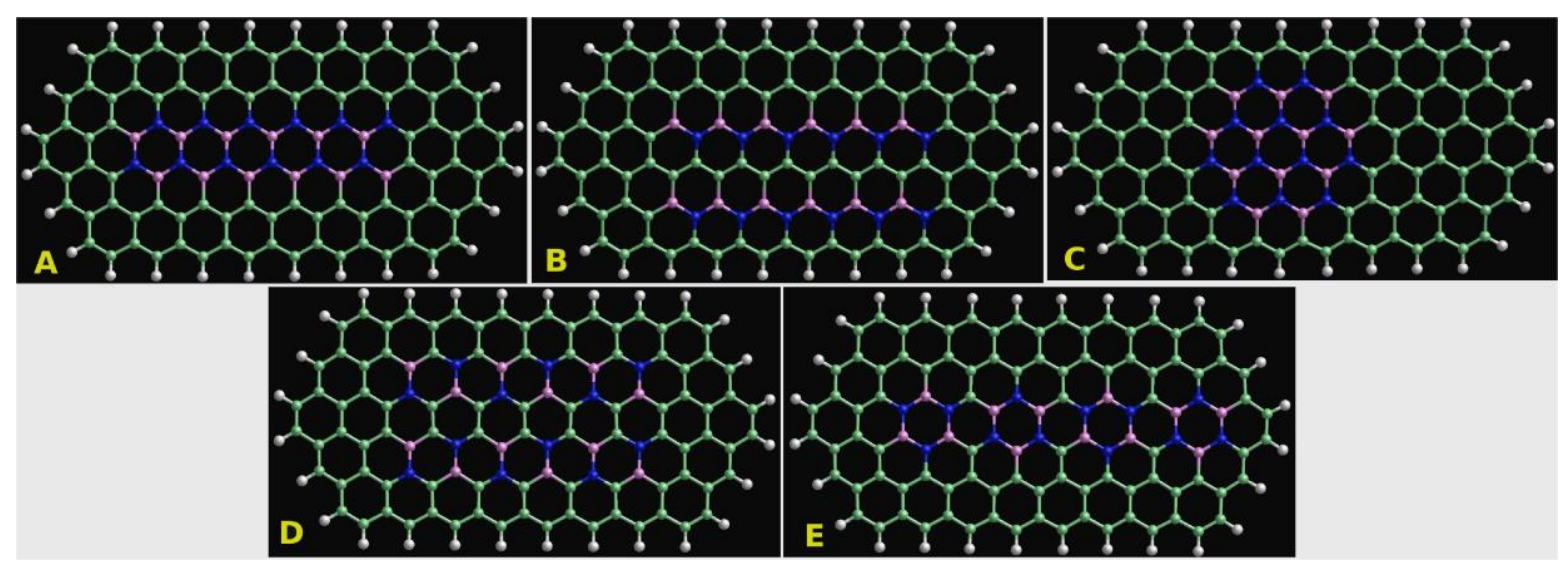

Figure 2. The doping of the graphene layer with 12BN compounds is shown. The carbon bonds are saturated with hydrogens at the borders. The insets from A to E differ in the positions of the binary compounds BN. In the insets $\mathrm{A}$ and $\mathrm{C}$ all the $\mathrm{BN}$ compounds are aggregated, instead, in the insets B, D and $\mathrm{E}$ the BN compounds form separated groups (color code: green $=$ carbon, white= hydrogen, blue= nitrogen, pink= boron).

Table 1. Electronic energies of the doped graphene layers. *

\begin{tabular}{|c|r|r|}
\hline System & Energy $(\mathrm{au})$ & \multicolumn{1}{|c|}{$\Delta \mathrm{E}(\mathrm{au})$} \\
\hline gp2BN & $-4359.218,200$ & -0.0 \\
\hline gp3BN & $-4366.089,793$ & $-10.360,961$ \\
\hline gp6BN & $-4369.579,161$ & $-20.720,220$ \\
\hline gp12BN (D) & $-4379.938,420$ & $-41.051,172$ \\
\hline gp12BN (B) & $-4400.269,372$ & $-41.396,780$ \\
\hline gp12BN (E) & $-4400.614,980$ & $-41.437,736$ \\
\hline gp12BN (A) & $-4400.655,936$ & $-41.586,770$ \\
\hline gp12BN (C) & $-4400.804,970$ & $-41.679,150$ \\
\hline & $-4400.897,350$ & -184.724 .919 \\
\hline BN8C & & $-191.762,636$ \\
\hline BN4C & $-4543.943,119$ & $-195.329,762$ \\
\hline BN2C & $-4550.980,836$ & $-198.898,444$ \\
\hline BN & $-4554.547,962$ & $-4558.116,644$ \\
\hline
\end{tabular}

*The nomenclature gpYBN indicates the graphene layer containing a number of $\mathrm{Y}$ molecules of the type BN, and $\mathrm{BNZC}$ indicates the $\mathrm{BN}$ layer containing a number of $\mathrm{Z}$ carbon atoms. The letters in parenthesis distinguish the different distributions of the BN dimers in the layer. The energies are shown from the less negative energy to the most negative energy. The energy differences denoted as $\Delta \mathrm{E}$ are presented, taking the energy of the bare graphene layer gp as the reference energy.

All the molecular systems are found energetically stable. The energy of the graphene layer is more negative by increasing the doping number of $\mathrm{BN}$ compounds. No linear relation is observed between the number of BN compounds and the energy change when we take the energy of the bare graphene layer as the reference energy of the systems. In the family of 12BN-doped layers, the structure with minimum energy is the structure with label C, followed by the A structure. These two have high connectivity of the BN molecules (like a planar cluster of BN compounds). Contrariwise, the structures $\mathrm{B}, \mathrm{D}$ and $\mathrm{E}$ have lower 
connectivity of $\mathrm{BN}$ molecules. Among these three structures, the E structure with $\mathrm{BN}$ closed rings shows the lowest energy. The structure B represents a kind of two unconnected linear doping and is higher in energy than the E structure. The D structure has the less negative energy in this family and represents a type of alternating doping (without reaching the doping of closed hexagons, as observed in the structures $\mathrm{C}, \mathrm{A}$ and $\mathrm{E}$ ). In principle, the structures with fully doped rings are the most stable structures, an important feature that may explain the energy difference between the bare graphene layer and the pure BN layer.

The bare BN layer is the system with the lowest electronic energy. The doping of the $\mathrm{BN}$ layer with carbon atoms makes the energy more positive and increasing with the number of carbon atoms. In principle, it is possible to mutate a graphene layer onto a BN layer with the replacement of CC pairs by $\mathrm{BN}$ binary compounds, and vice versa. The energy involved in such a transformation is $-198.898,444$ au for the type of layers of this work.

In Table 2, the average atom charge populations of $\mathrm{H}, \mathrm{B}, \mathrm{C}$ and $\mathrm{N}$ for the different types of layers are presented. They were computed according to the Mulliken scheme. The charge population of $\mathrm{H}$ is essentially indifferent to the type of layer studied here. The charge populations of $\mathrm{B}$, together with these of $\mathrm{N}$, are the ones with noticeable changes in both the graphene layers and the BN layers. The charges of B are positive, and these of $\mathrm{N}$ are negative. Meanwhile, the charge population of $\mathrm{C}$ changes signs in going from a graphene layer (where it is a small negative number in most instances) to a $\mathrm{BN}$ layer (where it takes positive values). The charge differences between $\mathrm{CC}$ pairs and the $\mathrm{BN}$ compounds is of interest since they point to local polarizations of the electrons. In this respect, we now analyze the electron density clouds and molecular electrostatic potentials in $3 \mathrm{D}$ space.

Table 2. Average atomic charge populations of different layers. *

\begin{tabular}{|c|c|c|c|c|}
\hline Layer & $\overline{\mathrm{q}}_{\mathrm{H}}$ & $\overline{\mathrm{q}}_{\mathrm{B}}$ & $\overline{\mathrm{q}}_{\mathrm{C}}$ & $\overline{\mathrm{q}}_{\mathrm{N}}$ \\
\hline Graphene & 0.10 & - & -0.02 & - \\
\hline gp2BN & 0.10 & 0.32 & -0.02 & -0.67 \\
\hline gp3BN & 0.10 & 0.45 & -0.02 & -0.63 \\
\hline gp6BN & 0.10 & 0.46 & -0.02 & -0.63 \\
\hline gp12BN (A) & 0.10 & 0.57 & -0.02 & -0.68 \\
\hline gp12BN (B) & 0.10 & 0.45 & -0.00 & -0.66 \\
\hline $\operatorname{gp12BN}(\mathrm{C})$ & 0.10 & 0.62 & -0.02 & -0.69 \\
\hline gp12BN (D) & 0.10 & 0.28 & 0.03 & -0.70 \\
\hline gp12BN (E) & 0.10 & 0.47 & -0.01 & -0.64 \\
\hline $\mathbf{B N}$ & 0.09 & 0.68 & - & -0.72 \\
\hline BN2C & 0.09 & 0.67 & 0.14 & -0.72 \\
\hline BN4C & 0.10 & 0.66 & 0.14 & -0.72 \\
\hline BN8C & 0.10 & 0.65 & 0.08 & -0.71 \\
\hline
\end{tabular}

*The nomenclature $\overline{\mathrm{q}}_{\mathrm{x}}$ indicates the average atomic charge of element X. On the other side, gpYBN indicates the graphene layer containing a number of $\mathrm{Y}$ molecules of the type BN, and BNZC indicates the BN layer containing a number of $\mathrm{Z}$ carbon atoms. The letters in parenthesis distinguish the different geometrical arrangements of the $\mathrm{BN}$ molecules in the layer. The units are electrons.

\footnotetext{
Volumetric properties: the electron density and molecular electrostatic potential

The pure graphene and pure $\mathrm{BN}$ layer are 2D crystal lattices and, therefore, show periodic forms of the electron density in their structures, except at the borders where the atoms linked to the terminal hydrogens show electron density changes. On the other hand, their hybridized layers are of more interest in this respect for analysis because, aside from energy differences, the doped layers exhibit electron density inhomogeneities that create local polarizations and, therefore, chemically active regions with possible
}

applications in catalysis. In order to characterize the doped graphene layers from such a perspective, the isosurfaces of both the electron density and the molecular electrostatic potential (MEP) are investigated. In Figure 3 the electron density maps of the systems gp2BN, gp3BN, gp12BN(A) and gp12BN $(\mathrm{C})$ are displayed. The doping with $\mathrm{BN}$ compounds breaks the uniform electron density of graphene, producing the depletion of electron density in some regions and the excess of electron density in other regions. 

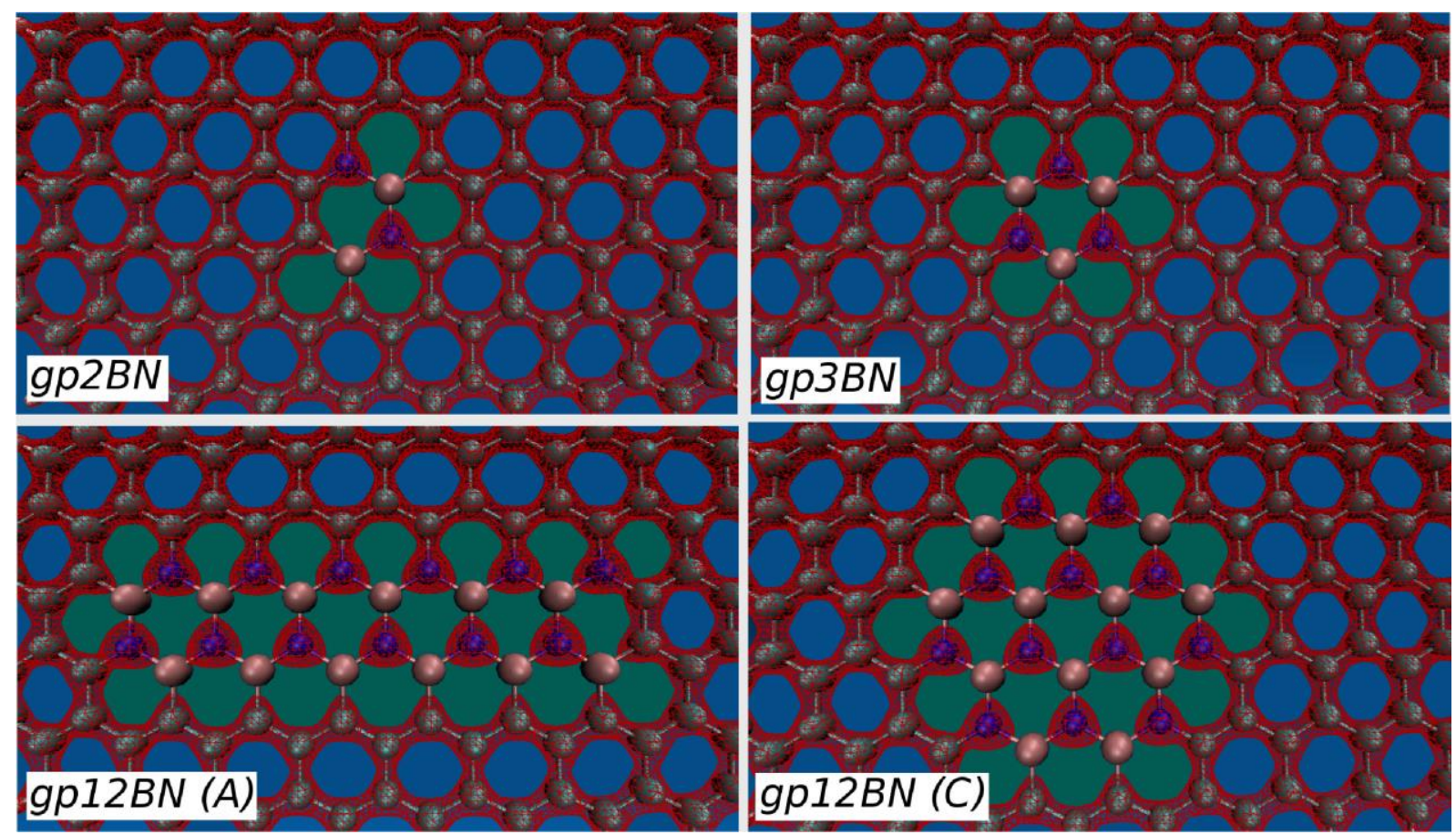

Figure 3. The electron densities of the hybrid layers gp2BN, gp3BN, gp12BN (A) and gp12BN (C) are depicted. The isosurface values of the density are $0.20 \mathrm{au}$, they are sketched in red color. The blue and green islands are empty spaces. The colors emphasize the shape differences of the electron densities due to the BN-doping compounds.

A similar situation is observed on the BN layers doped with carbon. In Figure 4 the electron density maps of the systems $\mathrm{BN} 2 \mathrm{C}, \mathrm{BN} 4 \mathrm{C}$ and $\mathrm{BN} 8 \mathrm{C}$ are shown.

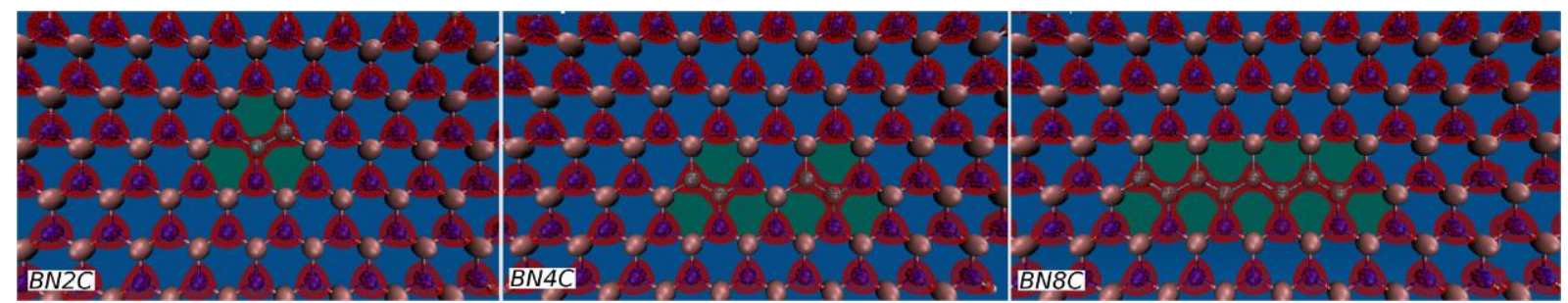

Figure 4. The electron densities of the hybrid layers BN2C, BN4C and BN8C are depicted. The isosurface values of the density are $0.20 \mathrm{au}$, they are sketched in red color. The blue and green islands are empty spaces. The colors emphasize the shape differences of the electron densities due to the C-doping compounds.

Again, the uniform electron density is perturbed by the presence of the carbon-atom doping. Nevertheless, the situation is different to the case of the doped graphene lattice. The reason is that in graphene the $\mathrm{BN}$ compounds are present in a background charge formed by carbon atoms, while in the doped boron-nitride layers the carbons are present in the background charge formed by $\mathrm{BN}$ molecules. The breaking of the uniform charge density in both the doped graphene and the doped boron-nitride meshes originates molecular electrostatic potentials, which are distributed according to the doping. As an example, the molecular electrostatic potential maps of the $\mathrm{BN} 2 \mathrm{C}$ and $\mathrm{BN} 4 \mathrm{C}$ layers are presented in the top insets of Figure 5. The doped graphene and doped boron-nitride layers are essentially flat, while the electron density and MEP are of volumetric type, in other words, they are 3D physical entities situated on a 2D material. In this context, the electron density irregularities and the MEPs are shown as clear protuberances of the 2D surface, making the volumetric surfaces easily noticeable to other charged compounds. Such features differ from those of other surfaces that are no strictly flat. 

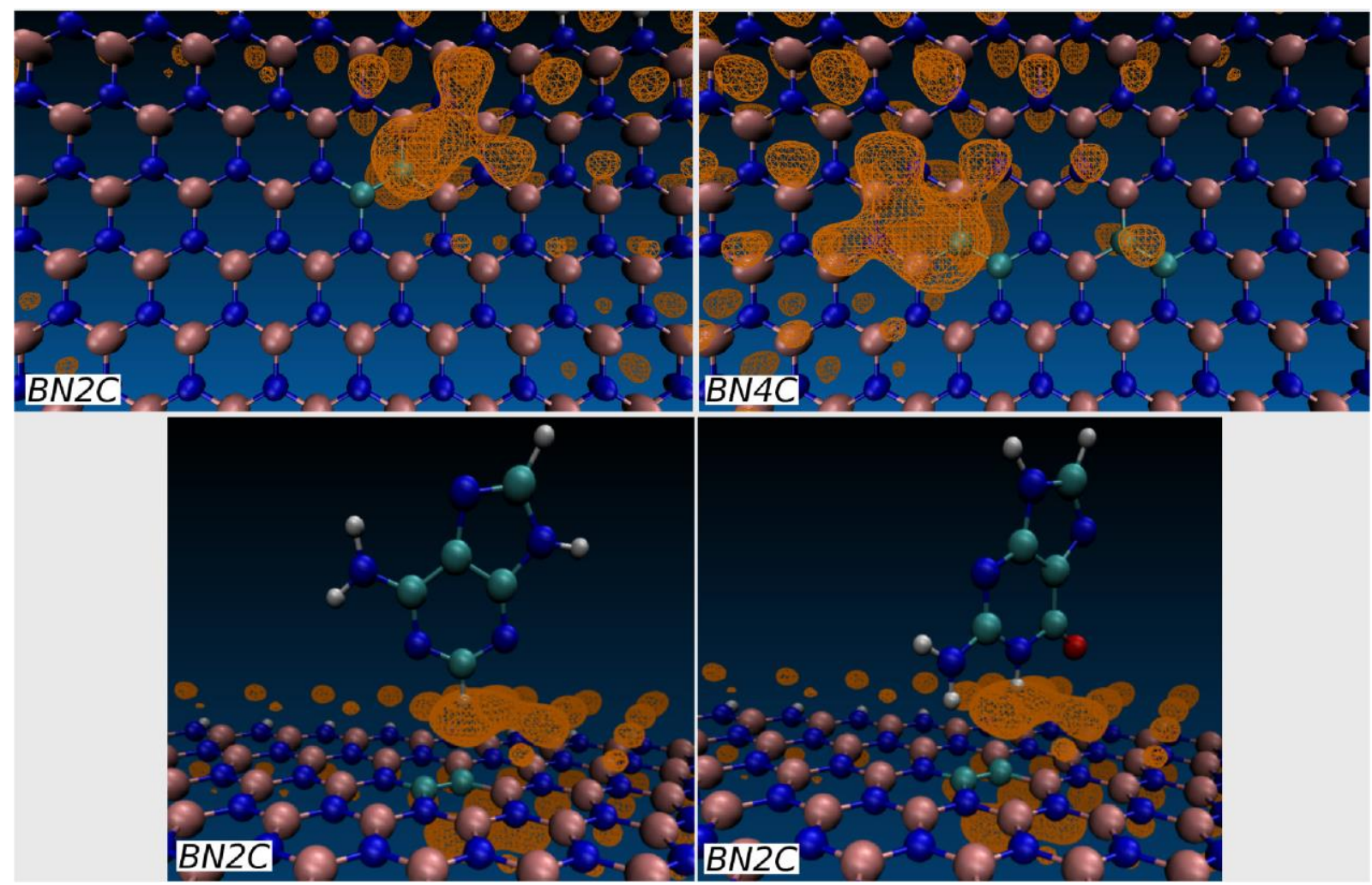

Figure 5. The molecular electrostatic potentials (MEPs) of the layers BN2C and BN4C are depicted in the first two insets at the top. The isosurface values of the MEPs are - $0.016 \mathrm{au}$, they are sketched in yellow-orange colors. The last two insets at the bottom show adenine (left side) and guanine (right side) on the BN2C lattices. For visualization purposes, the MEP of the first inset (BN2C) is superposed on the lattices at the bottom to show the locations of the hydrogens of adenine and guanine on the layer. Guanine is the only molecule adsorbed on the hybrid layer.

The MEPs of the C-doped boron-nitride lattices are found at the regions close to the carbon atoms (Figure 5). In this regard, the doped boron-nitride layers in principle present the opportunity on the design of MEPs by using C-doping at selected spatial regions of the layer. That is, it is possible to create MEPs by doping the boron-nitride layers with carbon atoms at chosen places and with the geometry of our interest. A similar situation but applied to the band gap has been reported in ${ }^{41}$, where it was shown that the band gap increases when the carbon atoms segregate into isolated, irregularly shaped graphene islands in the $\mathrm{BN}$ layers. Yet, for a given stoichiometry, the electronic and optical properties of a B-C-N layer can be tuned by growth kinetics. For h$\mathrm{BN}$ layers with $\mathrm{C}$ domains, it is equally possible to tune the h-BN band gap in the visible region ${ }^{42}$. Moreover, n-type and p-type semiconductors can be designed by controlling either the $\mathrm{C}-\mathrm{N}$ or $\mathrm{C}-\mathrm{B}$ bonds at the domain borders, with potential applications in photocatalysis and optoelectronic devices. Similar results have been observed on the band gap of multiwalled boron carbonitride nanotubes ${ }^{43}$. Still, it is important to emphasize that our results involve the tuning of the MEPs, rather than the energy band gaps, and such a tuning also shows potential applications on the docking of molecules on the BN layers, with consequent applications on chemical catalysis.
The adsorption of molecules on graphene-based materials is of interest and has been studied in different contexts. One of these has interfaced DNA with graphene oxide with the purpose of creating new sensors at the atomic level ${ }^{44}$. Here, it is shown that DNA is adsorbed on graphene oxide via П-П stacking and hydrogen bonding, simultaneously overcoming the electrostatic repulsions. The boron-nitride sheets have been also investigated with the goal of employing nanopores of atomic thickness. By simulating the dynamics of DNA, it has been demonstrated the possibility of detecting doublestranded DNA sequences from the ionic currents through the pore during the DNA translocation ${ }^{45}$. Our work differs from the previous studies by dealing with the molecular electrostatic potentials of C-doped BN layers, and the intention to trap biological molecules on top of such layers. In the two lower insets of Figure 5 we present a pair of nucleic acid bases of the DNA molecule on the surface of the boron-nitride lattice (the nucleic acid bases were energetically optimized on the substrate). The MEPs of the nucleic acid bases have been discussed in the context of biological processes elsewhere ${ }^{46}$. For our reference, the MEP of the BN2C lattice is superposed on such a lattice. As it is observed from the lower insets of Figure 5, the nucleic acid bases are linked to the substrate by hydrogens that are trapped in the electrostatic 
potential wells. Of course, more complex conformations of the $\mathrm{C}$ atoms on the boron-nitride sheet may be built for trapping other types of molecules.

The adsorption energy, for example of guanine, is computed in the form:

$$
\mathrm{E}(\mathrm{BN} 2 \mathrm{C}+\text { Guanine })-\mathrm{E}(\mathrm{BN} 2 \mathrm{C})-\mathrm{E}(\text { Guanine })
$$

The adsorption energy of guanine on the BN2C layer is close to $5 \mathrm{Kcal} / \mathrm{mole}$, and that of adenine is essentially zero.

\section{Vibrational analysis}

Our final purpose resides on briefly characterizing the dynamical aspects of the doped lattices. Smolyanitsky and Tewary studied the dynamic ripples on graphene by producing local harmonic out-ofplane excitations ${ }^{47}$. The authors demonstrated the possibility of tuning dynamic ripples by applying a local out-of-plane sinusoidal excitation to the graphene layer. Such work shows potential applications in mechanically controlling quantum dots. In our particular study on the dynamics of layers, we include both the doped graphene and doped boronnitride layers as well. The layers are mechanically perturbed by displacing a set of atoms located at the central part of the layer, and releasing such atoms. This is a mechanical perturbation that induces a vibration, and which propagates in every direction. The vibrational waves reach the borders of the lattice after some time, which depends on the lattice extension and composition. No periodic conditions are required in our case, since the interest is on investigating the effects of the doping elements on the propagation speed of the waves, an effect that is independent of the periodic conditions. In Table 3 the wave speeds of the different layers in their first vibration moments are provided. Such speeds are determined by measuring the time interval $\Delta t=t_{2}-t_{1}$, where $t_{1}$ is the initial moment of the release of the central atoms and $t_{2}$ is the instant of time when the wave reaches the most distant longitudinal borders (there are two borders of such a nature, one to the right and the other to the left).

Table 3. Wave speed in the left and right directions. *

\begin{tabular}{|c|c|c|}
\hline Layer & $\mathrm{V}_{\mathrm{l}}$ & $\mathrm{V}_{\mathrm{r}}$ \\
\hline Graphene & 0.1281 & 0.1268 \\
\hline gp2BN & 0.1243 & 0.1225 \\
\hline gp3BN & 0.1207 & 0.1231 \\
\hline gp6BN & 0.1201 & 0.1136 \\
\hline gp12BN (E) & 0.1294 & 0.1255 \\
\hline gp12BN (B) & 0.1198 & 0.1209 \\
\hline gp12BN (C) & 0.1158 & 0.0975 \\
\hline gp12BN (A) & 0.1050 & 0.1064 \\
\hline gp12BN (D) & 0.1004 & 0.1004 \\
\hline BN & 0.0668 & 0.0669 \\
\hline
\end{tabular}

*The nomenclature is the same as in Table 1. The symbols $\mathbf{v}$ and $\mathbf{v} \mathbf{r}$ indicate the wave speed to the left or right of the mechanical perturbation, respectively. The speed units are $\AA / f$ s.

The waves propagating on the graphene layers with low concentrations of $\mathrm{BN}$ compounds are in general the fastest waves. The wave speeds $v_{l}$ and $v_{r}$ are different when the doping elements are located either on the right side or the left side of the mechanical perturbation that generates the wave on the lattice. On the other hand, the wave speeds of the graphene layers with high concentrations of $\mathrm{BN}$ molecules are in general small and depend on the distribution of the $\mathrm{BN}$ compounds in the lattice, except for the gp12BN(E) hybrid layer, which shows a speed slightly bigger than that of pure graphene in the left direction. In the gp12BN(E) hybrid layer we have $\mathrm{BN}$ rings intercalated in linear form in the graphene layer and, on the other hand, the number of the $\mathrm{BN}$ rings is different in the left and right directions (Figure 2E). The intercalation and the directional asymmetry of the $\mathrm{BN}$ rings may be the cause of observing a large speed in the propagation of the perturbation with respect to that on pure graphene. In spite of that, the pure graphene shows fast wave speeds and the boron-nitride lattice slow ones (with an average speed ratio of 1.9). Apparently, the change of masses in replacing $\mathrm{CC}$ compounds with $\mathrm{BN}$ compounds, and the distribution of the doping elements (either to the left or right of the mechanical perturbation) are sufficiently important to imprint a difference in the wave propagation from the very first moments of the perturbation. In this respect, the mechanical wave speeds of graphene may be modulated by the presence of a number of $\mathrm{BN}$ compounds and their distribution in the graphene sheets.

\section{Conclusion}

The graphene (gp) and boron-nitride (BN) layers are physical systems with interesting properties in basic science and technology. The interconversion of such layers by doping a graphene layer with $\mathrm{BN}$ 
compounds, and a BN layer with carbon atoms leads to novel applications of graphene and boron-nitride compounds in addition to those reported in the literature, which have been particularly focused on optoelectronics ${ }^{48}$ Thus, in a complementary context, we have shown properties of physico-chemical and mechanical interest of the hybridized layers. The inhomogeneities of the electron density introduced by the doping elements originate polarization domains and, in turn, chemically active regions. The possibility of replacing $\mathrm{BN}$ compounds of the $\mathrm{BN}$ layer by $\mathrm{CC}$ atoms, and making them to describe a geometry of our interest, ${ }^{26}$ results on the creation of new molecular electrostatic potentials in the BN layer, capable to selectively adsorb either small or large molecules according to our needs. The redistribution of mass in replacing $\mathrm{CC}$ compounds with $\mathrm{BN}$ compounds, and vice versa, is sufficiently important to make a difference in the propagation of mechanical waves on the graphene and the boron-nitride layers, respectively. In consequence, the mechanical wave speeds of graphene (BN) may be modulated by both the presence of a specific number of $\mathrm{BN}$ (CC) compounds and their distribution in the graphene sheet. In principle, the properties of the hybridized layers elucidated in this investigation show potential applications in technology, and should be especially attractive to explore the docking of molecules, in addition to the investigation of new catalytic pathways and chemical processes.

\section{Acknowledgments}

This work is dedicated to Prof. Octavio Novaro, an excellent scientist and a wonderful person. The authors express gratitude to Dra. Laura Serkovic for reviewing this work. We also recognize DGTICUNAM and LAREC-IF for the supercomputing facilities, and the staff of the Institute of Physics (UNAM) for their valuable support. RS acknowledges financial support from the authorities of IF-UNAM under the project name PIIF-03, and project IN111918 from PAPIIT-DGAPA to perform this research.

\section{References}

1 - H. O. Pierson, Boron Nitride Composites by Chemical Vapor Deposition, J. Compos. Matter, 1975, 9, 228-240.

2 - R. Haubner, M. Wilhelm, R. Weissenbacher, B. Lux, Boron Nitrides - Properties, Synthesis and Applications, High Performance NonOxide Ceramics II, 2002, 102, 1-45.

3 - N. R. Glavin, C. Muratore, M. L. Jespersen, J. Hu, P. T. Hagerty, A. M. Hilton, A. T. Blake, C. A. Grabowski, M. F. Durstock, M. E. McConney, D. M. Hilgefort, T. S. Fisher, A. A. Voevodin, Amorphous Boron Nitride:

A Universal, Ultrathin Dielectric for 2D Nanoelectronics, Adv. Funct. Mater., 2016, 26, 2640-2647.
4 - L. Liu, Y. P Feng., Z. X Shen, Structural and Electronic Properties of h-BN, Phys. Rev. B, 2003, 68, 104102-8.

5 - A. H. Castro Neto, F. Guinea, N. M. R. Peres, K. S. Novoselov, A. K. Geim, The Electronic Properties of Graphene, Rev. Mod. Physics, 2009, 81, 109-162.

6 - J. C. Pacilé, Ç. Ö Meyer, A Girit,. Zettl, The Two-dimensional Phase of Boron Nitride: Fewatomic-layer Sheets and Suspended Membranes, Appl. Phys. Lett., 2008, 92, 133107-3.

7 - Z. Zheng, M. Cox, B. Li, Surface Modification of Hexagonal Boron Nitride Nanomaterials: a Review, J. Mater. Sci., 2018, 53, 66-99.

8 - M.F. Khan Shahil, A. Alexander Balandin, Thermal Properties of Graphene and Multilayer Graphene: Applications in Thermal Interface Materials, Solid State Comm., 2012, 152, 1331-1340.

9 - Eric Pop, V. Varshney, and Ajit K. Roy, Thermal Properties of Graphene: Fundamentals and Applications, MRS Bulletin, 2012, 37, 1273-1281.

10 - D. G. Papageorgiou, I. A. Kinloch, R. J. Young, Mechanical Properties of Graphene and Graphene-based Nanocomposites, Prog. Mater. Sci., 2017, 90, 75-127, https://doi.org/10.1016/j.pmatsci.2017.07.004.

11 - C. Lee, X. Wei, J. W. Kysar, J. Hone, Measurement of the Elastic Properties and Intrinsic Strength of Monolayer Graphene, Science, 2008, 321, 385-388.

12 - S. P. Koenig, N. G. Boddeti, M. L. Dunn, J. S. Bunch, Ultrastrong Adhesion of Graphene Membranes, Nat. Nanotechnol., 2011, 6, 543-546.

13 - Y. Wu, D. B. Farmer, F. Xia, P. Avouris, Graphene Electronics: Materials, Devices, and Circuits, Proc. IEEE, 2013, 101, 1620-1637, https://doi.org/10.1109/JPROC.2013.2260311.

14 - R. Raccichini, A. Varzi, S. Passerini and B. Scrosati, The Role of Graphene for Electrochemical Energy Storage, Nature Mater., 2015, 14, 271-279.

15 - Y. Yang, A. M. Asiri, Z. Tang, D. Du, Y. Lin, Graphene Based Materials for Biomedical Applications, Mater. Today, 2013, 16, 365-373.

16 - A. V. Eletskii, I. M. Iskandarova, A. A. Knizhnik, D. N. Krasikov, Graphene: Fabrication Methods and Thermophysical Properties, Phys.-Usp., 2011, 54, 227-258.

17 - P. R. Somani, S. P. Somani, M. Umeno, Planer Nano-graphenes from Camphor by CVD, Chem. Phys Lett., 2006, 430, 56-59.

18 - S. Gayathri, P. Jayabal, M. Kottaisamy, V. Ramakrishnan, Synthesis of Few Layer Graphene by Direct Exfoliation of Graphite and a Raman spectroscopic study, AIP Advances, 2014, 4, 027116-12, https://doi.org/10.1063/1.4866595. 
19 - (a) A. Kaniyoor, T. T. Baby, S. Ramaprabhu, Graphene Synthesis via Hydrogen Induced Low Temperature Exfoliation of Graphite Oxide, J. Mater. Chem., 2010, 20, 8467-8469.

(b) K. S. Novoselov, A. K. Geim, S. V. Morozov, D. Jiang, Y. Zhang, S. V. Dubonos, I. V. Grigorieva, A. A. Firsov, Electric Feld Effect in Atomically Thin Carbon Flms, Science, 2004, 306, 666-669.

20 - L. H. Li, J. Cervenka, K. Watanabe, T. Taniguchi, Strong Oxidation Resistance of Atomically Thin Boron Nitride Nanosheets, ACS Nano, 2014, 8, 1457-1462, https://pubs.acs.org/doi/pdf/10.1021/nn500059s

21 - S.-G. Xu, X.-T. Li, Y.-J. Zhao, J-H. Liao, W.-P. $\mathrm{Xu}, \mathrm{X} . \mathrm{-B}$. Yang, H. Xu, Two-Dimensional Semiconducting Boron Monolayers, J. Am. Chem. Soc., 2017, 139, 17233-17236.

22 - A. Falin, Q. Cai, E. J. G. Santos, D. Scullion, D. Qian, R. Zhang, Z. Yang, S. Huang, K.i Watanabe, T. Taniguchi, M. R. Barnett, Y. Chen, R. S. Ruoff, L. H. Li, Mechanical Properties of Atomically Thin Boron Nitride and the Role of Interlayer Interactions, Nat. Commun. 2017, 8, 15815.

23 - Q. Cai, A. Du, G. Gao, S. Mateti, B. C. C. Cowie, D. Qian, S. Zhang, Y. Lu, L. Fu, T. Taniguchi, S. Huang, Y. Chen, R. S. Ruoff, L. H. Li, 2D Nanomaterials: Molecule- induced Conformational Change in Boron Nitride Nanosheets with Enhanced Surface Adsorption, Adv. Funct. Mater. 2016, 26, 8356-8356.

24 - L. H. Li, Y. Chen, Atomically Thin Boron Nitride: Unique Properties and Applications, Adv. Funct. Mater. 2016, 26, 2594-2608.

25 - L. Ju, L. Wang, T. Cao, T. Taniguchi, K. Watanabe, S. G. Louie, F. Rana, J. Park, J. Hone, F. Wang, P. L. McEuen, Tunable Excitons in Bilayer Graphene, Science, 2017, 358, 907-910.

26 - L. Ci, L. Song, C. Jin, D. Jariwala, D. Wu, Y. Li, A. Srivastava, Z. F. Wang, K. Storr, L. Balicas, F. Liu, P. M. Ajayan, Atomic Layers of Hybridized Boron Nitride and Graphene Domains, Nat. Mater., 2010, 9, 430-435.

27 - R. Nascimento, J. da Rocha Martins, R. J. C. Batista, H. Chacham, Helio, Band Gaps of BN-Doped Graphene: Fluctuations, Trends, and Bounds, J. Phys. Chem. C, 2015, 119, 50555061.

28 - J. Li, P. Jin, W. Dai, C. Wang, R. Li, T. Wu, C. Tang, Excellent Performance for Water Purification Achieved by Activated Porous Boron Nitride Nanosheets, Mater. Chem. Phys., 2017, 196, 186-193.

29 - J. Wang, Fengcai Ma, M. Sun, Graphene, Hexagonal Boron Nitride, and Their Heterostructures: Properties and Applications, RSC Adv., 2017, 7, 16801-16822, http://dx.doi.org/10.1039/C7RA00260B. 30 - Y. Gong, G. Shi, Z. Zhang, W. Zhou, J. Jung, W. Gao, L. Ma, Y. Yang, S. Yang, G.
You, R. Vajtai, Q. Xu, A. H. MacDonald, B. I. Yakobson, J. Lou, Z. Liu, P. M. Ajayan, Direct Chemical Conversion of Graphene to Boronand Nitrogen- and Carbon-containing Atomic Layers, Nat. Comm., 2014, 5, 3193-8, http://dx.doi.org/10.1038/ncomms4193.

31 - Y. Chu, T. Ragab, C. Basaran, The Size Effect in Mechanical Properties of Finite-sized Graphene Nanoribbon, Comput. Mater. Sci., 2014, 81, 269-274.

32 - W. Li, L. Liang, S. Zhao, S. Zhang, J. Xue, Fabrication of Nanopores in a Graphene Sheet with Heavy Ions: A Molecular Dynamics study, J. Appl. Phys., 2013, 114, 234304-6.

33 - M. K. Rana, A. Chandra, Ab Initio and Classical Molecular Dynamics Studies of the Structural and Dynamical Behavior of Water Near a Hydrophobic Graphene Sheet, J. Chem. Phys., 2013, 138, 204702-9.

34 - A. V. Titov, I. S. Ufimtsev, N. Luehr, T. J. Martinez, Generating Efficient Quantum Chemistry Codes for Novel Architectures, J. Chem. Theory Comput., 2013, 9, 213-221.

35 - A. D. Becke, Density-functional Exchangeenergy Approximation with Correct Asymptotic Behavior, Phys. Rev. A, 1988, 38, 3098-3100.

36 - C. Lee, W. Yang, R. G. Parr, Development of the Colle-Salvetti Correlation-energy Formula into a Functional of the Electron Density, Phys. Rev. B, 1988, 37, 785-789.

37 - J. Kästner, J. M. Carr, T. W. Keal, W. Thiel, A. Wander, P. Sherwood, DL-FIND: An OpenSource Geometry Optimizer for Atomistic Simulations, J. Phys. Chem. A, 2009, 113, 11856-11865.

38 - M. P. Allen, D. J. Tildesley, Computer Simulation of Liquids, Oxford University Press: New York, 1989, pp. 78-82.

39 - I. S. Ufimtsev, T. J. Martinez, Quantum Chemistry on Graphical Processing Units. 3. Analytical Energy Gradients, Geometry Optimization, and First Principles Molecular Dynamics, J. Chem. Theory Comput., 2009, 5, 2619-2628.

40 - M. J. Frisch, G. W. Trucks, H. B. Schlegel, G. E. Scuseria, M. A. Robb, J. R. Cheeseman, G. Scalmani, V. Barone, G. A. Petersson, H. Nakatsuji, X. Li, M. Caricato, A. Marenich, J. Bloino, B. G. Janesko, R. Gomperts, B. Mennucci, H. P. Hratchian, J. V. Ortiz, A. F. Izmaylov, J. L. Sonnenberg, D. WilliamsYoung, F. Ding, F. Lipparini, F. Egidi, J. Goings, B. Peng, A. Petrone, T. Henderson, D. Ranasinghe, V. G. Zakrzewski, J. Gao, N.Rega, G. Zheng, W. Liang, M. Hada, M. Ehara, K. Toyota, R. Fukuda, J. Hasegawa, M. Ishida, T. Nakajima, Y. Honda, O. Kitao, H. Nakai, T. Vreven, K. Throssell, J. A. Montgomery, Jr., J. E. Peralta, F. Ogliaro, M. Bearpark, J. J. Heyd, E. Brothers, K. N. Kudin, V. N. Staroverov, T. Keith, 
R. Kobayashi, J. Normand, K. Raghavachari, A. Rendell, J. C. Burant, S. S. Iyengar, J. Tomasi, M. Cossi, J. M. Millam, M. Klene, C. Adamo, R. Cammi, J. W. Ochterski, R. L. Martin, K. Morokuma, O. Farkas, J. B.

Foresman, D. J. Fox, Gaussian 09, Revision D.01, Gaussian, Inc., Wallingford CT, 2016.

41 - J. da Rocha Martins, H. Chacham, Disorder and Segregation in B-C-N Graphene-Type Layers and Nanotubes: Tuning the Band Gap, ACS Nano, 2011, 5, 385-393.

42 - A. C. Bevilacqua, M. H. Köhler, S. Azevedo, R. J. Baierlea, Stability, Optical and Electronic Properties of Ultrathin h-BNC, Phys. Chem. Chem. Phys., 2017, 19, 5629-5636.

43 - S. Young Kim, J. Park, H. Chul Choi, J. Pyung Ahn, J. Qiang Hou, H. Seok Kang, X-ray Photoelectron Spectroscopy and First Principles Calculation of BCN Nanotubes, J. Am. Chem. Soc., 2007, 129, 1705-1716.
44 - B. Liu, S. Salgado, V. Maheshwari, J. Liu, DNA Adsorbed on Graphene and Graphene Oxide: Fundamental Interactions, Desorption and Applications, Curr. Opin. Colloid \& Interface Sci., 2016, 26, 41-49.

45 - Z. Gu, Y. Zhang, B. Luan, Z. Ruhong, DNA Translocation Through Single-layer Boron Nitride Nanopores, Soft Matter, 2016, 12, 817-823.

46 - R. Santamaria, G. Cocho, L. Corona, E. González, Molecular Electrostatic Potentials and Mulliken Charge Populations of DNA Mini-sequences, Chem. Phys., 1998, 227, 317-329.

47 - A. Smolyanitsky, V. K. Tewary, Manipulation of Graphene's Dynamic Ripples by Local Harmonic Out-of-plane Excitation, Nanotechnology, 2013, 24, 055701-8.

48 - A. Rubio, Hybridized Graphene: Nanoscale Patchworks, Nat. Mater., 2010, 9, 379-380. 